\title{
Novel Insights into Lossless AC and DC Power Flow
}

\author{
Florian Dörfler, Student Member, IEEE, and Francesco Bullo, Fellow, IEEE
}

\begin{abstract}
A central question in the analysis and operation of power networks is feasibility of the power flow equations subject to security constraints. For large-scale networks the solution of the nonlinear $\mathrm{AC}$ power flow equations can be constructed only numerically or approximated through the linear DC power flow. The latter serves as well-used approximation to obtain the phase angle differences near an acceptable operating point, but the accuracy of the DC approximation drops in a stressed grid. Here, we propose a modified DC approximation that applies to lossless networks with parametric uncertainties and with voltage magnitudes bounded within security constraints. We show that the phase angle differences can xbe well approximated by the solution to a set of linear and interval-valued equations reminiscent of the DC power flow equations. Our proposed approximation improves upon the standard DC approximation, is computationally attractive and provably exact for a broad range of network topologies and parameters. We validate the accuracy of our approximation through standard power network test cases with randomized power demand at the loads.
\end{abstract}

\section{INTRODUCTION}

$\mathbf{T}$ HE power flow equations are the basic mathematical model underpinning the analysis, operation and planning of power networks. A central question is "under which conditions on the network parameters and topology and the load and generation profile, does there exist an optimal [1], stable [2][4], and robust [5]-[9] synchronous operating point". A more general concern is whether the power flow equations admit any solution [10], [11] or an existing solution vanishes in a saddle node bifurcation [12], [13]. Various security indices have been proposed to quantify the robustness margin of a particular operating condition [14]. The assessment of an acceptable synchronous operating point and the quantification of its robustness margin will become more and more important in an increasingly complex, volatile, and stressed grid.

In general, analytic answers to the above questions are known only for radial (acyclic) networks [1], [10], [14], only loose conditions are known for arbitrary networks [5], [7], [8], and various puzzling examples are known [6]. For more complex networks, the above questions can be answered only through numerical computation [15]. For large-scale networks, a numerical treatment of the coupled active and reactive power flow equations quickly becomes computationally expensive and unfeasible. Hence, the DC power flow is the only viable alternative [16] in large-scale systems. Furthermore, for many applications, such as real-time contingency screening, security assessment, and transmission planning, various parametric scenarios have to be examined which adds upon the computational complexity, even in the DC case. One of the well-known drawbacks of the DC power flow is that it is an accurate

This material is based in part upon work supported by NSF grant CPS1135819.

Florian Dörfler and Francesco Bullo are with the Center for Control, Dynamical Systems and Computation, University of California at Santa Barbara, Santa Barbara, CA 93106. Email: \{dorfler, bullo\}@engineering.ucsb.edu approximation only near an operating point with small phase angle differences and nearly unit voltage magnitudes.

In this article, we leverage recent results in synchronization theory [17] to improve upon the standard DC power flow approximation. In particular, we are interested in the phase angle differences obtained as solutions to the power flow equations. We show that the angle differences can be well approximated by the solution to a set of linear and intervalvalued equations reminiscent of the DC power flow equations.

As a first contribution, we propose a modified DC approximation to the lossless active power flow equations with constant voltage magnitudes. Our proposed approximation is computationally attractive, and is either provably exact or satisfied with inequality and high accuracy for a broad range of network topologies and parameters, including the cases of small angle differences, bipolar angles, acyclic topologies, complete topologies, small cycles, and combinations of these networks. We validate our proposed approximation with a set of randomized power network test cases, and we demonstrate that it improves upon the accuracy obtained by the standard DC approximation. As a second contribution, we construct a set of interval-valued DC power flow equations to overapproximate voltage variations and parametric uncertainties in the coupled active and reactive power flow equations. We show that solutions of the power flow equations can be efficiently bounded by extremal solutions of the interval-valued DC power flow equations. This interval-valued approach together with our modified DC approximation is a powerful tool for contingency screening and uncertainty analysis, and we validate its accuracy for a set of randomized test cases.

The paper is organized as follows. Section II reviews the AC power flow equations and their DC approximation. Section III presents our modified DC approximation for constant and variable voltages and parameters. Finally, Section IV concludes the paper and suggests a few interesting applications.

\section{REVIEW OF THE AC AND DC POWER FLOW}

\section{A. Review of the Lossless AC Power Flow Equations}

For our purposes, a power network is a connected, undirected, and complex-weighted graph $G(\mathcal{V}, \mathcal{E}, Y)$ (or equivalently a linear circuit) with node set (or buses) $\mathcal{V}=\{1, \ldots, n\}$, edge set (or branches) $\mathcal{E} \subset \mathcal{V} \times \mathcal{V}$, and symmetric weights (or admittances) $Y_{i j}=Y_{j i} \in \mathbb{C}$ for every undirected edge $\{i, j\} \in \mathcal{E}$. With every node $i \in \mathcal{V}$, we associate the power injection $S_{i}=P_{i}+\sqrt{-1} \cdot Q_{i} \in \mathbb{C}$ and the phasor variable $V_{i} e^{\sqrt{-1} \cdot \theta_{i}}$ corresponding to the magnitude and the phase shift of a harmonic solution to the AC circuit equations. In a highvoltage transmission network, the lines are typically lossless and inductive, that is, $Y_{i j} \approx-\sqrt{-1} \cdot B_{i j}$, where $B_{i j}>0$ is the susceptance of the line $\{i, j\} \in \mathcal{E}$. For a lossless network, 
the power flow equations are obtained in polar coordinates as

$$
\begin{aligned}
P_{i} & =\sum_{j=1}^{n} B_{i j} V_{i} V_{j} \sin \left(\theta_{i}-\theta_{j}\right), \quad i \in\{1, \ldots, n\}, \\
Q_{i} & =-\sum_{j=1}^{n} B_{i j} V_{i} V_{j} \cos \left(\theta_{i}-\theta_{j}\right), \quad i \in\{1, \ldots, n\} .
\end{aligned}
$$

The variables of the power flow equations (1) are $\left(\theta_{i}, V_{i}\right)$ for PQ-buses and $\left(\theta_{i}, Q_{i}\right)$ for PV-buses. The power flow equations (1) constitute a set of $2 n$ coupled equations with trigonometric and polynomial nonlinearities, and the solution space admits a rich and complex phenomenology [6], [13]. For practical power network operation, the solutions $\left(\theta^{*}, V^{*}\right)$ of the power flow equations (1) of interest satisfy the security constraints

$$
\begin{array}{ll}
\left|\theta_{i}^{*}-\theta_{j}^{*}\right| \leq \gamma_{i j}, & \{i, j\} \in \mathcal{E}, \\
0<\underline{V_{i}} \leq V_{i}^{*} \leq \overline{V_{i}}, & i \in\{1, \ldots, n\} .
\end{array}
$$

The security constraints limit the branch power flows and prevent instabilities, and they typically range within $\gamma_{i j} \in$ $\left[5^{\circ}, 25^{\circ}\right], V_{i} \approx 0.95$, and $\overline{V_{i}} \approx 1.05$ in per unit (p.u.) system. In this paper, we are primarily interested in angular differences over transmission lines, $\theta_{i}-\theta_{j}$ for $\{i, j\} \in \mathcal{E}$, which govern the active flow and are responsible for rotor angle synchronism.

We do not assume the (mathematically convenient but physically doubtful) presence of a slack bus. Instead, we maintain the rotational symmetry of the power flow, that is, if $\theta^{*}$ is a solution to the power flow equations (1), then a rigid rotation of all angles yields another solution. A necessary condition for solvability of the power flow equations (1) is $\sum_{i=1}^{n} P_{i}=0$, that is, that the active power injections must be balanced ${ }^{1}$.

\section{B. Review of the DC Power Flow Equations}

Due to local voltage control, due to adequate reactive power support, or due to the classic power flow decoupling assumption, all voltage magnitudes $V_{i}$ in the active power flow equations (1a) are of roughly unit value near an acceptable operating point. Under the assumption $V_{i}=1$ for all $i \in\{1, \ldots, n\}$, the active power flow equations (1a) reduce to

$$
P_{i}=\sum_{j=1}^{n} B_{i j} \sin \left(\theta_{i}-\theta_{j}\right), \quad i \in\{1, \ldots, n\} .
$$

Under nominal operating conditions, the angular distances over transmission lines are "small", and the equations (3) are commonly approximated by the DC power flow equations [16]:

$$
P_{i}=\sum_{j=1}^{n} B_{i j},\left(\delta_{i}-\delta_{j}\right), \quad i \in\{1, \ldots, n\} .
$$

Since the DC power flow (4) equations are sparse and linear, they can be solved efficiently with reliable and non-iterative methods and with minimal network data. The DC power flow (4) can be conveniently rewritten in vector form as

$$
P=L \delta,
$$

where $\delta=\left(\delta_{1}, \ldots, \delta_{n}\right), P=\left(P_{1}, \ldots, P_{n}\right)$, and $L=\sqrt{-1}$. $Y \in \mathbb{R}^{n \times n}$ is the Laplacian matrix (or susceptance matrix) defined component-wise as $L_{i j}=-B_{i j}$ for off-diagonal elements and $L_{i i}=\sum_{j=1}^{n} B_{i j}$ for diagonal elements. The solution to the DC power flow equations (5) is uniquely determined up

${ }^{1}$ If $\sum_{i=1}^{n} P_{i} \neq 0$, then by summing over all equations (1a), we arrive at the contradiction $\sum_{i=1}^{n} P_{i}=0$, and the equations (1) are inconsistent. to a rigid translation of all angles $\delta$ as $\delta^{*}=L^{\dagger} P$, where $L^{\dagger}$ is the Moore-Penrose pseudo-inverse of the Laplacian matrix $L$.

A standard Taylor series expansion yields that the phase differences obtained by the DC power flow (4) approximate those obtained by the AC power flow (3) for sufficiently small angular differences $\left|\theta_{i}^{*}-\theta_{j}^{*}\right| \ll 1$ for all $\{i, j\} \in \mathcal{E}$ :

DC approximation: For the active power flow equations (3) with solution $\theta^{*}$ and the DC power flow equations (4) with solution $\delta^{*}=L^{\dagger} P$, consider the approximation

$$
\theta_{i}^{*}-\theta_{j}^{*} \approx \delta_{i}^{*}-\delta_{j}^{*} \quad \text { for all }\{i, j\} \in \mathcal{E}
$$

The DC approximation (6) is computationally attractive, reasonably accurate, and well used in practice [16], but it may perform poorly in volatile operating conditions. Unfortunately, even when the accuracy of the DC approximation (6) is doubtful, there is often no viable alternative in computationally intensive applications such as large-scale power flow optimization, contingency screening, and transmission planning.

\section{AnAlytic And Statistical Results}

In this section, we leverage our recent results [17] to improve upon the DC approximation. The three subsections consider the cases of constant voltage magnitudes, variable voltage magnitudes, and uncertain network parameters.

\section{A. Analytic Results for Constant Voltage Magnitudes}

In this subsection, we assume constant voltage magnitudes, and we improve upon the standard approximation of the active power flow equations (3) via the DC power flow equations (4). We propose the following modified DC approximation:

Modified DC approximation: For the active power flow equations (3) with solution $\theta^{*}$ and the DC power flow (4) with solution $\delta^{*}=L^{\dagger} P$, consider the approximation

$$
\theta_{i}^{*}-\theta_{j}^{*} \approx \arcsin \left(\delta_{i}^{*}-\delta_{j}^{*}\right) \quad \text { for all }\{i, j\} \in \mathcal{E} \text {. }
$$

The approximation (7) can be nicely motivated in a cyclefree network. In this case, a direct calculation shows that the approximation (7) is exact since we can recover $\theta^{*}$ from $\delta^{*}$ :

$$
\begin{aligned}
P_{i} & =\sum_{j=1}^{n} B_{i j} \sin \left(\theta_{i}^{*}-\theta_{j}^{*}\right) \\
& =\sum_{j=1}^{n} B_{i j} \sin \left(\arcsin \left(\delta_{i}^{*}-\delta_{j}^{*}\right)\right) \\
& =\sum_{j=1}^{n} B_{i j}\left(\delta_{i}^{*}-\delta_{j}^{*}\right)=P_{i}, \quad i \in\{1, \ldots, n\} .
\end{aligned}
$$

On the other hand, if the network features cycles, then all angular differences $\theta_{i}^{*}-\theta_{j}^{*}$ (respectively $\delta_{i}^{*}-\delta_{j}^{*}$ ) around a cycle must sum to zero, whereas the corresponding terms $\arcsin \left(\delta_{i}^{*}-\delta_{j}^{*}\right)$ do not necessarily sum to zero. In this case, the approximation (7) does not hold with exact equality. Finally, notice that the proposed approximation (7) has no additional computational cost compared to the DC approximation (6).

Whereas the standard DC power flow approximation (6) is a first-order approximation for infinitesimally small difference 
angles, our modified DC approximation (7) can be derived as a first-order approximation of the solution $\theta^{*}$ in the cut-set space of the network, where cycle constraints are neglected, see the appendix of [17]. We showed in [17] that for a broad range of topologies and parameters, the approximation (7) is exact and holds with equality, or it holds at least with inequality, that is,

$$
\left|\theta_{i}^{*}-\theta_{j}^{*}\right| \leq \arcsin \left(\left|\delta_{i}^{*}-\delta_{j}^{*}\right|\right) \quad \text { for all }\{i, j\} \in \mathcal{E} .
$$

In particular, for networks with extremal topologies and parameters, we have that the approximation (7) holds

1) with equality for the sparsest graphs (acyclic),

2) with inequality for the densest graphs (complete graphs with homogeneous weights),

$3)$ with equality for sufficiently aligned angles $\delta_{i}^{*} \approx \delta_{j}^{*}$ for all $\{i, j\} \in \mathcal{E}$ (corresponding to small active power flows $\left|\sin \left(\theta_{i}^{*}-\theta_{j}^{*}\right)\right| \ll 1$ for all $\left.\{i, j\} \in \mathcal{E}\right)$, and

4) with equality for bipolar angles $\delta_{i}^{*} \in\{+c,-c\}$ for some $c \in[0,1 / 2]$ and $i \in\{1, \ldots, n\}$ (corresponding to bipolar active power flows $\sin \left(\theta_{i}^{*}-\theta_{j}\right) \in\{0, \pm \sin (2 c)\}$ for all $\{i, j\} \in \mathcal{E}$ ).

Besides the extremal graphs 1)-4), we also showed analytically that the approximation (7) holds

5) with inequality for small cycle graphs with cycles of lengths 3 and 4,

6) with equality for arbitrary cycle graphs with symmetric angles, where the histogram of the entries of $\delta^{*}$ is symmetric, and

7) with inequality for arbitrary one-connected combinations of weighted graphs each satisfying properties 1)-6).

We also showed that the inequality condition (8) is statistically correct [17] for a broad range of random networks of various sizes, with different random graph topologies, with randomized weights $B_{i j}$, and with randomized power injections $P_{i}$. The precise mathematical statements can be found in [17].

It is well-known that many results and conjectures about the solutions $\theta^{*}$ to the power flow equations (3) fail to hold for sufficiently large isolated cycles [1], [6], [10], [14]. Indeed, we were able to construct a family of counter-examples consisting of cycles of length 5 or larger, where inequality (8) fails with an error of order 0.05 . We remark that these examples are at the boundary of the admissible parameter space (equations (3) are feasible only with extremal power flows $\sin \left(\theta_{i}^{*}-\theta_{j}^{*}\right)= \pm 1$ for some lines) and do not seem to occur in generic networks.

\section{B. Numerical Results for Constant Voltage Magnitudes}

Obviously, real-world power networks are carefully engineered, they cannot be represented by random graph models, and they do not match the extremal graphs in 1) - 7). Hence, to validate our approximation (7), we test its accuracy for various power system test cases. In particular, we use the following test cases found in found in [18]: 9 bus system by Chow, IEEE 14, IEEE 30, IEEE 39 (New England), IEEE 57, IEEE 118, and the Polish 2383 bus system (Winter 1999-2000 configuration).

In order to obtain a large pool of representative samples under volatile operating conditions, we start from the above test cases (with optimal generation and fixed voltage magnitudes) and modify them as follows: 1) We assume fluctuating demand and randomize $50 \%$ of all loads to deviate from their forecast with Gaussian statistics (with standard deviation 0.3 p.u.). 2) We assume that the grid is penetrated by renewables, and we randomize $33 \%$ of all generating units to deviate from the nominal generation with Gaussian statistics (with standard deviation 0.3 p.u.). 3) To mitigate these fluctuations, we assume that the grid is equipped with $10 \%$ fast-ramping generation and $10 \%$ controllable loads, and the active power imbalance (caused by fluctuating demand and generation) is uniformly dispatched among these adjustable sources/sinks.

Since the above modifications are quite severe, they result in heavily stressed grids with possibly unrealistically large power flows. Thus, if a sample results in unrealistically high difference angles, $\left|\theta_{i}^{*}-\theta_{j}^{*}\right| \geq \pi / 4=45^{\circ}$ for some $\{i, j\} \in \mathcal{E}$, then it is discarded, and a new sample is constructed. The statistical properties of the samples are reported in the second and third column of Table I. It can be observed that the sampled networks are stressed and feature large angle differences.

For each test case, we construct 1000 random realizations of the scenario 1), 2), and 3) described above, we numerically solve the active power flow equations (1a) and the DC power flow equations (4). To evaluate the performance of the standard DC approximation (6) and our proposed modified DC approximation (7), we define the worst-case errors ${ }^{2}$

$$
\begin{aligned}
e_{\mathrm{DC}} & =\max _{\{i, j\} \in \mathcal{E}}\left|\left(\theta_{i}^{*}-\theta_{j}^{*}\right)-\left(\delta_{i}^{*}-\delta_{j}^{*}\right)\right|, \\
e_{\text {mod-DC }} & =\max _{\{i, j\} \in \mathcal{E}}\left|\left(\theta_{i}^{*}-\theta_{j}^{*}\right)-\arcsin \left(\delta_{i}^{*}-\delta_{j}^{*}\right)\right| .
\end{aligned}
$$

For each sample, we calculate the worst-case errors (9) and then evaluate their statistics over all samples. Our findings are reported in Table I. Observe that the proposed approximation (7) is always at least as accurate as the standard DC approximation (6). Both errors are comparable for the IEEE 57 and the Polish grid. For all other cases the proposed approximation (7) is more accurate, sometimes even by an entire order of magnitude. An exemplary error distribution is shown in Fig. 1.

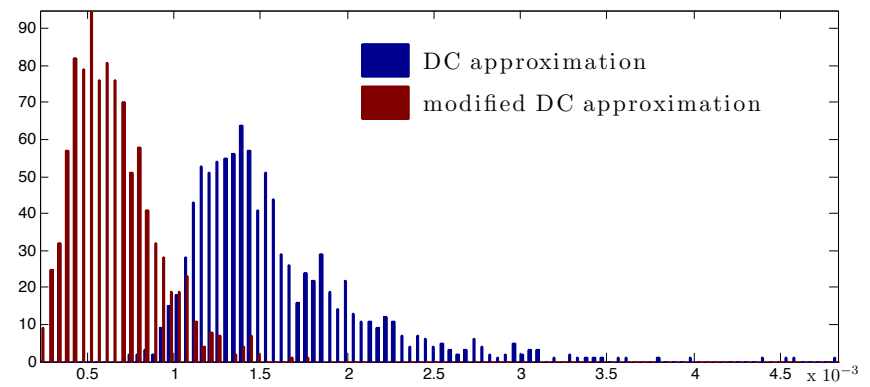

Fig. 1. Histogram of the worst-case errors (9) for the IEEE 118 bus system. Since the angular differences are limited to at most $\pi / 4$, the DC approximation (6) is quite accurate on average, but the error distribution has long and fat tails. In comparison, the proposed modified DC approximation (7) is significantly more accurate and the error distribution has short and thin tails.

\section{Analytic Results for Variable Voltage Magnitudes}

Even under nominal operating conditions, the voltage magnitudes are not constant, they may vary slowly, or they may be known only with a certain degree of accuracy. To extend the

\footnotetext{
${ }^{2}$ Since for most lines the angular differences are small (see the mean values in Table I), the two approximations (6) and (7) are identical for these lines. Hence, we examine worst-case $\ell_{\infty}$ errors rather than $\ell_{1}$ or $\ell_{2}$ error norms.
} 
TABLE I

Properties of power system test cases with randomized loads and generation, statistics of the errors (9), and validation of the approximations (6) and (7).

\begin{tabular}{|c|c|c|c|c|c|c|c|c|}
\hline randomized test case & mean & $\max$ & \multicolumn{2}{|c|}{ mean accuracy } & \multicolumn{2}{|c|}{ variance accuracy } & \multicolumn{2}{|c|}{ worst accuracy } \\
\hline (all u & $\max _{\{i, j\} \in \mathcal{E}}\left|\theta_{i}^{*}-\theta_{j}^{*}\right|$ & $\max _{\{i, j\} \in \mathcal{E}} \mid \theta_{i}^{*}-\theta_{j}^{*}$ & $\underset{1000 \text { it. }}{\operatorname{mean}} e_{\mathrm{DC}}$ & $\begin{array}{l}\text { mean } \\
1000 \text { it. }\end{array}$ & $\underset{1000 \text { it. }}{\operatorname{var}} e_{\mathrm{DC}}$ & $\operatorname{var}_{1000 \text { it. }} e$ & $\max _{1000 \text { it. }} e_{\text {DC }}$ & $\max _{1000 \text { it. }} e$ \\
\hline Chow 9 bus & 0.1294 & 0.3208 & $4.101 \cdot 10^{-4}$ & $5.243 \cdot 10^{-5}$ & $1.698 \cdot 10^{-7}$ & $6.627 \cdot 10^{-9}$ & $4.460 \cdot 10^{-3}$ & $1.067 \cdot 10^{-3}$ \\
\hline IEEE 14 bus $\mathrm{sy}$ & 0.1641 & 0.3382 & $.559 \cdot 10^{-4}$ & $2.749 \cdot 10^{-4}$ & $1.655 \cdot 10^{-7}$ & $2.242 \cdot 10^{-8}$ & $4.972 \cdot 10^{-3}$ & $1.523 \cdot 10^{-3}$ \\
\hline IEEE 30 bus system & 0.1489 & 0.5123 & $7.457 \cdot 10^{-4}$ & $1.652 \cdot 10^{-4}$ & $1.741 \cdot 10^{-6}$ & $7.765 \cdot 10^{-8}$ & $2.212 \cdot 10^{-2}$ & $3.506 \cdot 10^{-3}$ \\
\hline New England 39 & 0.1683 & 0.1941 & $7.592 \cdot 10^{-4}$ & $1.572 \cdot 10^{-4}$ & $4.708 \cdot 10^{-9}$ & $7.790 \cdot 10^{-10}$ & $1.055 \cdot 10^{-3}$ & $2.753 \cdot 10^{-4}$ \\
\hline IEEE 57 bus sy & 0.2782 & 0.7632 & $1.392 \cdot 10^{-2}$ & $1.556 \cdot 10^{-2}$ & $2.413 \cdot 10^{-3}$ & $3.531 \cdot 10^{-3}$ & $6.575 \cdot 10^{-1}$ & $6.585 \cdot 10^{-1}$ \\
\hline IEEE 118 bus system & 2318 & 31 & $1.590 \cdot 10^{-3}$ & $6.479 \cdot 10^{-4}$ & $2.638 \cdot 10^{-7}$ & $5.749 \cdot 10^{-8}$ & $4.840 \cdot 10^{-3}$ & $1.769 \cdot 10^{-3}$ \\
\hline olish 2383 bus system & 0.2524 & 0.3878 & $1.592 \cdot 10^{-3}$ & $1.404 \cdot 10^{-3}$ & $6.002 \cdot 10^{-7}$ & $6.192 \cdot 10^{-7}$ & $1.423 \cdot 10^{-2}$ & $1.449 \cdot 10^{-}$ \\
\hline
\end{tabular}

results of the previous subsection to the unmodeled reactive power flow equations (1b), we need to account for variable voltage magnitudes $V_{i}$. In order to do so, we assume that each voltage magnitude $V_{i}$ can vary within pre-specified upper and lower bounds as in (2b). Given the bounds (2b), the maximal active power flows $a_{i j} \triangleq B_{i j} V_{i} V_{j}$ in (1) are bounded within $\underline{a_{i j}}=B_{i j} \cdot \underline{V_{i}} \cdot \underline{V_{j}}$ and $\overline{a_{i j}}=B_{i j} \cdot \overline{V_{i}} \cdot \overline{V_{j}}, \quad\{i, j\} \in \mathcal{E}$. Next, we over-approximate the coupled power flow equations (1) by the interval-valued active power flow equations

$$
P_{i}=\sum_{j=1}^{n} a_{i j} \sin \left(\theta_{i}-\theta_{j}\right), \quad i \in\{1, \ldots, n\},
$$

where $a_{i j} \in\left[a_{j}, \overline{a_{j}}\right]$. Notice that every solution $\theta^{*}$ resulting from the coupled active and reactive power flow equations (1) can also be produced by the interval-valued active power flow (10). Accordingly, define the interval-valued DC power flow

$$
P_{i}=\sum_{j=1}^{n} a_{i j}\left(\delta_{i}-\delta_{j}\right), \quad i \in\{1, \ldots, n\} .
$$

Based on classic circuit theory and interval-valued analysis, we showed in [17] that extremal solutions $\delta^{*}$ of the interval-valued equations (11) are obtained for extremal parameter values, i.e,

$$
\begin{aligned}
\min _{a_{i j} \in\left[\underline{a_{j}}, \overline{a_{j}}\right]}\left(\delta_{i}^{*}-\delta_{j}^{*}\right) & =\min _{a_{i j} \in\left\{\underline{a_{j}}, \overline{a_{j}}\right\}}\left(\delta_{i}^{*}-\delta_{j}^{*}\right) \triangleq p_{i j}^{\min }, \\
\max _{a_{i j} \in\left[\underline{a_{j}}, \overline{\left.a_{j}\right]}\right.}\left(\delta_{i}^{*}-\delta_{j}^{*}\right) & =\max _{a_{i j} \in\left\{\underline{a_{j}}, \overline{a_{j}}\right\}}\left(\delta_{i}^{*}-\delta_{j}^{*}\right) \triangleq p_{i j}^{\max } .
\end{aligned}
$$

Based on the bounds (12) and the results in Subsection III-A, we propose the following approximation:

Modified interval-valued DC approximation: For the power flow equations (1) with solution $\left(\theta^{*}, V^{*}\right)$ satisfying the voltage bounds (2b) and the interval-valued DC power flow equations (4) with solutions $\delta^{*}$ satisfying the bounds (12), consider for all $\{i, j\} \in \mathcal{E}$ the approximation

$$
\arcsin \left(p_{i j}^{\min }\right) \leq\left|\theta_{i}^{*}-\theta_{j}^{*}\right| \leq \arcsin \left(p_{i j}^{\max }\right) .
$$

The exact evaluation of the approximation (13) is computationally expensive since all vertices of the parameter-space $a_{i j} \in\left[a_{j}, \overline{a_{j}}\right]$ need to be sampled in a combinatorial way to obtain the quantities (12a)-(12b). In practice, we found that randomized Monte Carlo sampling methods or simplex-type algorithms perform well in practice and quickly deliver an accurate estimate of the quantities (12a)-(12b). For certain topologies, such as acyclic ones, it is also possible to analytically determine the maximizing vertices beforehand, and the combinatorial quantities (12a)-(12b) reduce to scalar ones.
As a final remark, once the critical quantities (12a)-(12b) are computed, the proposed approximation (13) can also be replaced by the standard DC power flow approximation (6) as

$$
p_{i j}^{\min } \leq\left|\theta_{i}^{*}-\theta_{j}^{*}\right| \leq p_{i j}^{\max } .
$$

\section{Analytic Results for Parametric Network Uncertainties}

The results in the previous subsection can also be extended to parametric uncertainties, such as unknown or uncertain active power demands or line characteristics. Hence, we consider the power flow equations (1) subject to the variations

$$
\begin{aligned}
\underline{P_{i}} & \leq P_{i} \leq \overline{P_{i}}, \quad i \in\{1, \ldots, n\}, \\
0<\underline{B_{i j}} & \leq B_{i j} \leq \overline{B_{i j}}, \quad\{i, j\} \in \mathcal{E} .
\end{aligned}
$$

If the voltage magnitudes satisfy the bounds (2b), then the maximal active power flows $a_{i j} \triangleq B_{i j} V_{i} V_{j}$ are bounded within

$\underline{a_{i j}}=\underline{B_{i j}} \cdot \underline{V_{i}} \cdot \underline{V_{j}}$ and $\overline{a_{i j}}=\overline{B_{i j}} \cdot \overline{V_{i}} \cdot \overline{V_{j}}, \quad\{i, j\} \in \mathcal{E}$.

Again, we consider the interval-valued active power flow (10) and the associated interval-valued DC power flow (11) subject to the voltage bounds (2b) and the parametric variations (10) and (15). Analogously, we then obtain the extreme values

$$
\begin{aligned}
& \min \left(\delta_{i}^{*}-\delta_{j}^{*}\right)=\quad \min \left(\delta_{i}^{*}-\delta_{j}^{*}\right) \triangleq p_{i j}^{\min }, \\
& a_{i j} \in\left[\underline{a_{j}}, \overline{a_{j}}\right], P_{i} \in\left[\underline{P_{i}}, \overline{P_{i}}\right] \quad a_{i j} \in\left\{\underline{a_{j}}, \overline{a_{j}}\right\}, P_{i} \in\left\{\underline{P_{i}}, \overline{P_{i}}\right\} \\
& \max \left(\delta_{i}^{*}-\delta_{j}^{*}\right)=\quad \max \left(\delta_{i}^{*}-\delta_{j}^{*}\right) \triangleq p_{i j}^{\max } . \\
& a_{i j} \in\left[\underline{a_{j}}, \overline{a_{j}}\right], P_{i} \in\left[\underline{P_{i}}, \overline{P_{i}}\right] \quad a_{i j} \in\left\{\underline{a_{j}}, \overline{a_{j}}\right\}, P_{i} \in\left\{\underline{P_{i}}, \overline{P_{i}}\right\}
\end{aligned}
$$

With these estimates we can apply either of the approximations (13) or (14) to obtain bounds on the angular differences.

\section{E. Numerical Results for Variable Voltages and Injections}

Similar to Subsection III-B, we evaluate the accuracy of the proposed approximation (13) through randomized power network test cases. We consider the same set of test cases as in Subsection III-B. In comparison to Subsection III-B, we do not assume fixed voltage magnitudes and rather solve the coupled active and reactive power flow equations (1). We make the following modifications for each test case: 1) We randomize $50 \%$ of all loads with Gaussian statistics with nominal reactive power demands as mean and standard deviation 0.2 p.u.. The active power demand is not modified. 2) For the modified loads, we resolve optimal generator dispatch problem. Thus, we obtain 1000 samples for each test case, where each sample has different voltage magnitudes at all buses, different reactive power demand at the loads, and different injections at the generators. The statistical properties of these samples are 
TABLE II

Properties of power system test cases with randomized loads and optimal generation, statistics of the errors (17), and validation of the approximation (13).

\begin{tabular}{|c|c|c|c|c|c|c|c|}
\hline $\begin{array}{l}\text { randomized test case } \\
\text { (all units are in }[\mathrm{rad}] \text { ) }\end{array}$ & $\begin{array}{c}\text { mean } \\
\max _{1 \in\{1, \ldots, n\}} P_{i}-\min _{1000 \text { it. }} P_{i}\end{array}$ & \begin{tabular}{|c|}
$\max$ \\
$i \in\{1, \ldots, n\}$ \\
$\max _{1000 \text { it. }} P_{i}-\min _{1000 \text { it. }} P_{i}$
\end{tabular} & $\begin{array}{c}\text { mean } \\
i \in\{1, \ldots, n\} \\
\max _{1000 \text { it. }} V_{i}-\min _{1000 \text { it. }} V_{i}\end{array}$ & 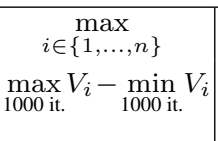 & $\begin{array}{c}\text { mean accuracy } \\
\text { mean } e_{\text {mod-DC }}^{(i, j)} \\
1000 \text { it. },\{i, j\} \in \mathcal{E}\end{array}$ & $\begin{array}{l}\text { variance accuracy } \\
\operatorname{var} e_{\text {mod-DC }}^{(i, j)} \\
1000 \text { it., }\{i, j\} \in \mathcal{E}\end{array}$ & \begin{tabular}{|c|} 
worst accuracy \\
$\max e_{\text {mod-DC }}^{(i, j)}$ \\
1000 it., $\{i, j\} \in \mathcal{E}$
\end{tabular} \\
\hline Chow 9 bus system & $8.751 \cdot 10^{-9}$ & $3.887 \cdot 10^{-8}$ & 0.0525 & 0.0812 & $2.063 \cdot 10^{-2}$ & $9.523 \cdot 10^{-14}$ & $1.007 \cdot 10^{-1}$ \\
\hline IEEE 14 bus system & 0.4797 & 2.6232 & 0.2000 & 0.2735 & $1.124 \cdot 10^{-1}$ & $1.332 \cdot 10^{-8}$ & $4.084 \cdot 10^{-1}$ \\
\hline IEEE 30 bus system & 0.1491 & 0.9908 & 0.3335 & 0.3823 & $5.434 \cdot 10^{-2}$ & $5.451 \cdot 10^{-8}$ & $2.947 \cdot 10^{-1}$ \\
\hline New England 39 & 0.3787 & 4.3915 & 0.0305 & 0.0517 & $5.079 \cdot 10^{-2}$ & $1.238 \cdot 10^{-9}$ & $1.661 \cdot 10^{-1}$ \\
\hline IEEE 57 bus system & 0.3623 & 5.7588 & 0.4928 & 0.5988 & $1.656 \cdot 10^{-1}$ & $3.233 \cdot 10^{-7}$ & $8.757 \cdot 10^{-1}$ \\
\hline IEEE 118 bus system & 0.4703 & 5.2713 & 0.0592 & 0.1178 & $1.023 \cdot 10^{-1}$ & $1.391 \cdot 10^{-12}$ & $6.351 \cdot 10^{-1}$ \\
\hline Polish 2383 bus system & 0.1269 & 24.000 & 0.5352 & 0.7506 & $2.573 \cdot 10^{-2}$ & $1.342 \cdot 10^{-8}$ & $4.836 \cdot 10^{-1}$ \\
\hline
\end{tabular}

reported in Table II. Observe that the injections and voltage magnitudes fluctuate severely, especially for large networks.

We use these 1000 samples to numerically evaluate the accuracy of the approximations (13) and (14) for variable voltages and parameter values, where $p_{i j}^{\min }$ and $p_{i j}^{\max }$ are computed as in (16). Given the solution $\left(\theta^{*}, V^{*}\right)$ of the power flow equations (1), define the approximation errors for each branch:

$$
\begin{aligned}
& e_{\bmod -\mathrm{DC}}^{(i, j)}=\max \left\{\left|\left(\theta_{i}^{*}-\theta_{j}^{*}\right)-\arcsin \left(p_{i j}^{\min }\right)\right|,\right. \\
&\left.\left|\left(\theta_{i}^{*}-\theta_{j}^{*}\right)-\arcsin \left(p_{i j}^{\max }\right)\right|\right\}, \\
& e_{\mathrm{DC}}^{(i, j)}=\max \left\{\left|\left(\theta_{i}^{*}-\theta_{j}^{*}\right)-p_{i j}^{\min }\right|,\left|\left(\theta_{i}^{*}-\theta_{j}^{*}\right)-p_{i j}^{\max }\right|\right\} .
\end{aligned}
$$

For each branch $\{i, j\} \in \mathcal{E}$ and each sample, we calculate the errors (17), and then evaluate their statistics. Our findings are reported in Table II and an exemplary error distribution is shown in Fig. 2. Notice that Table II contains only the approximation error $e_{\text {mod-DC }}$ and not $e_{\mathrm{DC}}$. The reason is that both errors are identical within an accuracy of $10^{-2}$ since all networks have small phase differences. Observe that, despite severe variations in generator power injections and voltage magnitudes, the proposed approximation (13) is extremely accurate in the mean. However, the worst-case values may be up to a factor 10 larger and can be of the same magnitude as the phase differences in Table II. We conclude that the proposed approximation (13) is very accurate on average, but for certain rare values of network parameters and voltage magnitudes its accuracy drops and the results are conservative.

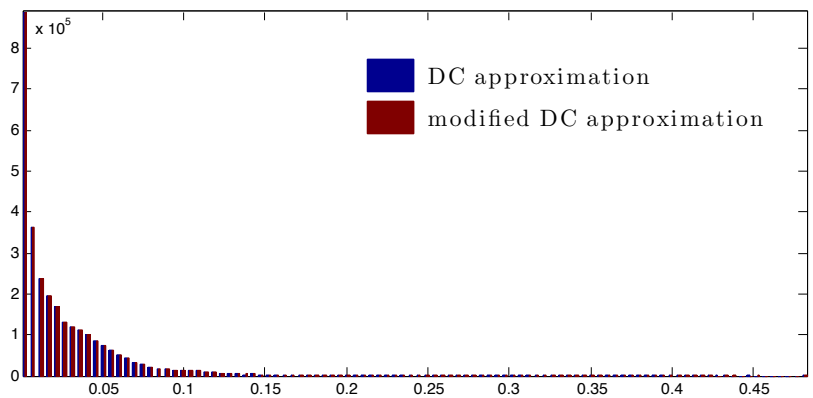

Fig. 2. Histogram of all errors (17) for 1000 samples of the Polish grid. Since the sampled networks have small angular differences, we obtain $\arcsin \left(\delta_{i}^{*}-\right.$ $\left.\delta_{j}^{*}\right) \approx \delta_{i}^{*}-\delta_{j}^{*}$, and the two approximation errors in (17) are nearly identical.

\section{CONCLUSions}

In this paper, we proposed a modified DC power flow approximation, which is not necessarily restricted to small angles, constant voltage magnitudes, and certain parameters. Our results are provably exact for various interesting cases, and we validated their accuracy with randomized power system test cases. We envision that our modified DC approximation can be used in various applications, such as security constrained power flow optimization, transmission line planning, real-time contingency screening, and online security assessment.

\section{REFERENCES}

[1] J. Lavaei, D. Tse, and B. Zhang, "Geometry of power flows in tree networks," in IEEE Power \& Energy Society General Meeting, San Diego, CA, USA, 2012, to appear.

[2] K. S. Chandrashekhar and D. J. Hill, "Cutset stability criterion for power systems using a structure-preserving model," International Journal of Electrical Power \& Energy Systems, vol. 8, no. 3, pp. 146-157, 1986.

[3] S. Sastry and P. Varaiya, "Hierarchical stability and alert state steering control of interconnected power systems," IEEE Transactions on Circuits and Systems, vol. 27, no. 11, pp. 1102-1112, 1980.

[4] F. Dörfler and F. Bullo, "Synchronization and transient stability in power networks and non-uniform Kuramoto oscillators," SIAM Journal on Control and Optimization, vol. 50, no. 3, pp. 1616-1642, 2012.

[5] M. Ilić, "Network theoretic conditions for existence and uniqueness of steady state solutions to electric power circuits," in IEEE International Symposium on Circuits and Systems, San Diego, CA, USA, May 1992, pp. 2821-2828.

[6] A. Araposthatis, S. Sastry, and P. Varaiya, "Analysis of power-flow equation," International Journal of Electrical Power \& Energy Systems, vol. 3, no. 3, pp. 115-126, 1981.

[7] F. Wu and S. Kumagai, "Steady-state security regions of power systems," IEEE Transactions on Circuits and Systems, vol. 29, no. 11, pp. 703711,1982

[8] F. F. Wu and S. Kumagai, Limits on Power Injections for Power Flow Equations to Have Secure Solutions. Electronics Research Laboratory, College of Engineering, University of California, 1980.

[9] J. Jarjis and F. D. Galiana, "Quantitative analysis of steady state stability in power networks," IEEE Transactions on Power Apparatus and Systems, vol. 100, no. 1, pp. 318-326, 1981.

[10] H. Chiang and M. Baran, "On the existence and uniqueness of load flow solution for radial distribution power networks," IEEE Transactions on Circuits and Systems, vol. 37, no. 3, pp. 410-416, 1990.

[11] B. C. Lesieutre, P. W. Sauer, and M. A. Pai, "Existence of solutions for the network/load equations in power systems," IEEE Transactions on Circuits and Systems I: Fundamental Theory and Applications, vol. 46, no. 8, pp. 1003-1011, 1999.

[12] S. Grijalva and P. W. Sauer, "A necessary condition for power flow Jacobian singularity based on branch complex flows," IEEE Transactions on Circuits and Systems I: Fundamental Theory and Applications, vol. 52, no. 7, pp. 1406-1413, 2005.

[13] I. Dobson, "Observations on the geometry of saddle node bifurcation and voltage collapse in electrical power systems," IEEE Transactions on Circuits and Systems I: Fundamental Theory and Applications, vol. 39, no. 3, pp. 240-243, 1992

[14] M. M. Hamada, M. A. A. Wahab, and N. G. A. Hemdan, "Simple and efficient method for steady-state voltage stability assessment of radial distribution systems," Electric Power Systems Research, vol. 80, no. 2 pp. 152-160, 2010.

[15] F. D. Galiana, "Analytical investigation of the power flow equations," in American Control Conference, San Francisco, CA, USA, Jun. 1983, pp. 411-415.

[16] B. Stott, J. Jardim, and O. Alsac, "DC power flow revisited," IEEE Transactions on Power Systems, vol. 24, no. 3, pp. 1290-1300, 2009.

[17] F. Dörfler, M. Chertkov, and F. Bullo, "Synchronization in complex oscillator networks and smart grids," Proceedings of the National Academy of Sciences, May 2012, to appear.

[18] R. D. Zimmerman, C. E. Murillo-Sánchez, and D. Gan, "MATPOWER: Steady-state operations, planning, and analysis tools for power systems research and education," IEEE Transactions on Power Systems, vol. 26, no. 1 , pp. $12-19,2011$ 